\title{
Comparison of three different techniques for histological tooth preparation
}

\author{
Nurullah Keklikoglu, Sevtap Akinci
}

Division of Basic Sciences, Department of Histology and Embryology, Faculty of Dentistry, Istanbul University, Istanbul, Turkey

\begin{abstract}
The histological processing of teeth is highly complicated because of containing both mineralized hard tissues and soft tissues. Depending on the type of decalcification agents used in processing, mild-to-severe deterioration in the tissue structure and inadequacies on clear staining of details by the histological stain may be observed. This study aims to compare the histological staining differences in the preparations from decalcified and undecalcified tooth roots by three different embedding materials and techniques. Following extraction, human single-rooted teeth crowns were cut off and roots were placed in $10 \%$ buffered neutral formalin. After fixation, roots were divided into two groups. One part of samples was decalcified in formic acid solution and the other was remained undecalcified. Decalcified roots were embedded in paraffin and glycol methacrylate (GMA)-based resin and undecalcified roots were embedded in methyl methacrylate (MMA)-based resin. Sections from all groups were stained with hematoxylin and eosin. The groups were compared in terms of general staining, brightness, density, density of the base stain, general morphology of cells, nuclear/cytoplasmic contrast, distinguishability of pulp, odontoblast layer, predentin and dentin, preservation and traceability of dentinal tubule. In the preparations which were embedded into the MMA-based embedding material, an output lower than the paraffin group but higher than the GMA-embedded group was provided. As a result, the best histological detail was obtained from the decalcified, paraffin-embedded sections. (Folia Histochemica et Cytobiologica 2013, Vol. 51, No. 4, 286-291)
\end{abstract}

Key words: tooth histology; uncalcified tissue; demineralization; embedding; paraffin; methyl methacrylate; glycol methacrylate

\section{Introduction}

Human teeth and bones consist of hard and soft tissues. The mineralised tissue with the most calcium content in the human body is tooth enamel with a ratio of $96-98 \%$ [1,2], followed by the dentin layer of tooth with $70 \%[3,4]$, while the mineralisation ratio of cementum is close to that of the bone and is 50\% [5].

Samples used in tooth and bone studies are very difficult for good preparation to further analyses as they contain high levels of mineralised hard tissues and soft tissues such as dental pulp and bone marrow. Therefore, well-customised methods are required for

Correspondence address: N. Keklikoglu, Division of Basic Sciences, Department of Histology and Embryology, Faculty of Dentistry, Istanbul University, Capa, Istanbul, Turkey tel.: +90 2124142020 30221;

e-mail: nkeklik@istanbul.edu.tr histological studies of these tissues. A common method to obtain sections from hard tissues to obtain histological sections is to soften them by demineralisation. However, acid-containing substances used for tissue decalcification and the time elapsed for the performing this procedure may negatively affect the histological structure. Depending on the type of acid used, mild -to-severe deterioration of the tissue structure and inadequate visualisation of details by the histological staining may be observed. For example, enamel, a tissue with very high calcium content, becomes almost completely lost during the decalcification procedure. Nevertheless, the techniques developed have minimised the negative effects of long-term fixation [6] and decalcification on the results of the histological and immunohistochemical staining of hard tissues $[7,8]$. However, there is a constant demand on the development of better techniques to perform quick, safe, repeatable and easy investigations of hard tissues' extracellular matrix, cells or intracellular specific struc- 
tures [9]. Research on the improvement of bones and teeth processing techniques has been continuing [10].

Given the fact that calcified areas may be seen even in the dental pulp from time to time, the reliability of the histological and pathological diagnoses upon the decalcified and paraffin-embedded tooth sections will always be controversial. Currently, it is possible to make histological preparations from undecalcified teeth by the use of specific methods and tools, which are, however, more expensive and difficult from classical histological techniques. These relatively new preparation techniques of hard tissues provide some important advantages, unfortunately, they require laboratories with highly specialized equipment, qualified personnel, advanced facilities and safety standards and they are also very demanding $[11,12]$. Therefore, these techniques cannot be easily accessed and performed by most of the researchers in this field. Moreover, as they are primarily developed by aiming at the preservation of bone tissue, these techniques may not be eligible for the preservation of the teeth, which contain harder tissues than the bone.

Paraffin is the standard embedding medium for more specific methods such as enzyme and immunohistochemistry in the hard tissues but it can be used only in the demineralised tissues and does not produce good results in trabecular structures [13]. It has been argued that paraffin, used as an embedding material for demineralised tooth histology, is the best method to analyse the histological structure of the tooth [14] due to its low price, ease of use and the flexibility of staining procedures to be applied on the paraffin-embedded tissues [15]. Nevertheless, paraffin is not hard enough for the processing of undecalcified tooth and bone. For such structures, hard embedding materials such as glycol methacrylate (GMA; (2-hydroxyethyl)-methacrylate, [HEMA]) or methyl methacrylate (MMA) are required [15]. Although using GMA-based embedding medium for soft tissues is thought to provide a better morphological analysis as it prevents some technical artefacts observed in paraffin-embedded sections, it has been reported that staining may be poor in resin sections [16].

The aim of this study was to compare the quality of routine staining with hematoxylin and eosin $(\mathrm{H} \& \mathrm{E})$ after embedding decalcified tooth roots into paraffin and GMA-based embedding material, and undecalcified hard tooth roots into a MMA-based embedding material, polymerised at $-20^{\circ} \mathrm{C}$.

\section{Material and methods}

The study protocol was approved by Istanbul University Istanbul Medical Faculty Ethical Committee (2008-3006). In this study, single-rooted non-decayed teeth extracted for prosthetic, orthodontic or surgical indications were used. Since the dental enamel will completely dissolve and lost in the decalcified group, not allowing comparison with undecalcified enamel, the crown parts of the tooth were not used. After crowns of total 40 permanent teeth from 20 female and 20 male patients between $18-50$ years old were resected, roots were fixed in $10 \%$ buffered neutral formalin. Roots at an equal number from female and male patients were divided into two groups ( 10 female and 10 male teeth in each group). The roots in one group were decalcified in $8 \%$ formic acid solutions, which were replaced every day for 1 to 2 weeks, with the endpoint being determined radiographically. Following the completion of decalcification, $1 / 3$ apical part of each root were extracted and excluded from the study. The remaining part of the roots were divided in two transversely and from the middle, and then blocked by embedding into paraffin and a GMA-based embedding material. The roots in the other group were blocked by directly embedding into a MMA-based embedding material without prior decalcification.

Paraffin embedding procedure. After the decalcification procedure was completed, the tissues were washed in distilled water, changed for 5-6 times in order to remove the acid solution. The washed tissues were transferred into the series of alcohol at increasing ratios for dehydration procedure. Following dehydration, clearing procedure was performed on the tissues by holding in xylene solution. The tissues cleared with xylene were transferred into soft paraffin (melting point $46-48^{\circ} \mathrm{C}$ ) and hard paraffin (melting point $56-58^{\circ} \mathrm{C}$ ) baths respectively. Then, they were blocked by embedding into hard paraffin.

Sections of $7 \mu \mathrm{m}$ thickness were taken from the blocked tissues by rotary microtome (Leitz, Wetzlar, Germany) and $\mathrm{H} \& \mathrm{E}$ staining was performed. After the H\&E staining, the sections were dehydrated and coverslipped using mounting media (Entellan Neu, Merck, Darmstadt, Germany).

\section{Embedding procedure with GMA-based embedding ma-} terial. After the decalcification procedure, the tissues washed with water were transferred into the series of alcohol at increasing ratios for dehydration procedure. As the GMA-based embedding material used (7022-18 500 Leica Historesin Embedding Kit, Heidelberg, Germany) contained 6-8\% water, absolute alcohol was not applied at the end of the dehydration procedure. The tissues were hold at the refrigerator for 24 hours, inside an infiltration solution prepared by mixing basic resin and activator $(50 \mathrm{~mL}$ liquid basic resin $+0.5 \mathrm{~g}$ activator powder) in a magnetic mixer. After the infiltration was completed, tooth roots were embedded into the embedding medium prepared $(15 \mathrm{~mL}$ infiltration solution $+1 \mathrm{~mL}$ hardener). Polymerisation of the parts was conducted within 1-2 hours at room temperature. Sections of $7 \mu \mathrm{m}$ thickness were taken from each of the polymerised tissues by automatic microtome (Leica SM 2500, Leica, 
Table 1. Results from light microscopic assessment of tooth root preparations embedded into paraffin, glycol methacrylate (GMA)- and methyl methacrylate (MMA)-based embedding materials and stained with hematoxylin and eosin (H\&E)

\begin{tabular}{|l|l|l|l|l|}
\hline \multirow{2}{*}{} & \multirow{2}{*}{ Scale } & Embedding material & \multicolumn{3}{|l|}{} \\
\cline { 3 - 5 } & & Parafin & GMA & MMA \\
\hline \multirow{3}{*}{ HE } & $-\mathrm{n}(\%)$ & $0(0)$ & $16(80)$ & $15(75)$ \\
\cline { 2 - 5 } & $+\mathrm{n}(\%)$ & $10(50)$ & $4(20)$ & $3(15)$ \\
\cline { 2 - 5 } & $++\mathrm{n}(\%)$ & $6(30)$ & $0(0)$ & $2(10)$ \\
\cline { 2 - 5 } & $+++\mathrm{n}(\%)$ & $4(20)$ & $0(0)$ & $0(0)$ \\
\hline
\end{tabular}

Scoring system: - negative (unacceptable); + average (acceptable); ++ good; +++ optimal

Germany). These sections were transferred onto slides from the distilled water bath and dried.

The tissues on the slides were taken into the series of alcohol in turn at decreasing ratios for redehydration procedure. Sections hold in each alcohol solution for 5-10 minutes were finally transferred into distilled water (5-10 minutes). Then, the tissue sections were stained with $\mathrm{H} \& \mathrm{E}$. After the staining procedure, the sections were dehydrated and coverslipped using mounting media (Entellan Neu).

Embedding procedure with MMA-based embedding material. For mineralised (undecalcified) tooth roots, a highly hard MMA-based embedding material (Technovit 9100 New; Heraeus Kulzer GmbH, Wehrheim, Germany) was used.

After the fixation procedure, the washed tissues were transferred into the series of alcohol at increasing ratios for dehydration procedure. After dehydration, the tissues were hold in a solution prepared for the preinfiltration procedure (200 $\mathrm{mL}$ basic solution $+1 \mathrm{~g}$ hardener 1$)$ at room temperature for 2 hours. After the preinfiltration was completed, the tissues were hold in infiltration solution $(250 \mathrm{~mL}$ basic solution + powder $20 \mathrm{~g}+1 \mathrm{~g}$ hardener 1$)$ at $+4{ }^{\circ} \mathrm{C}$ for 24 hours. The tissues were covered with a mixture prepared for polymerisation ( 9 volumes of stock solution A [ $80 \mathrm{~g}$ powder $+400 \mathrm{~mL}$ stabilised basic solution $+4 \mathrm{~g}$ hardener 1$]+1$ volume of stock solution $\mathrm{B}$ [ $4 \mathrm{~mL}$ hardener $2+30 \mathrm{~mL}$ stabilised basic solution $+2 \mathrm{~mL}$ regulator]) and hold at $+4^{\circ} \mathrm{C}$ or 10 minutes. Then, the blocks were placed as closed in the freezer at $-20^{\circ} \mathrm{C}$ for 24 hours.

Sections of $7 \mu \mathrm{m}$ thickness were taken from each of the polymerised tissues by automatic microtome (Leica SM 2500, Leica, Germany). For deplastication, the sections were taken into xylene $(2 \times 20$ minutes), 2-MEA (2-methoxyethylacetate) $(3 \times 30$ minutes $)$, acetone $(2 \times 10$ minutes $)$ and distilled water $(2 \times 5$ minutes $)$ solutions, respectively, before the staining. After removal of the resin, H\&E staining was performed.

Light microscopic assessment. 10 sections from each one of the 20 blocks in each three groups (total 600 sections) were analysed. The criteria used for the assessments were:
- General staining, brightness, density, density of the base stain,

- General morphology of cells, nuclear/cytoplasmic contrast,

- Distinguishability of pulp, odontoblast layer, predentin and dentin,

- Preservation and traceability of dentinal tubules.

Rating was performed semi-quantitatively according to these criteria, using a 4-degree scale: -negative (unacceptable); + average (acceptable); + + good; ++ +optimal.

Statistical analyses. Pearson chi-square test was applied to the data obtained and statistical significance margin was accepted as p value $<0.05$.

\section{Results}

Numerical data from the assessments performed are provided in Table 1. The best result in terms of the assessment criteria - general staining, brightness, density, density of the base stain, general morphology of cells, nuclear/cytoplasmic contrast, distinguishability of pulp, odontoblast layer, predentin and dentin, preservation and traceability of dentinal tubule - was observed with the preparations which were decalcified in formic acid, embedded into paraffin and stained with $\mathrm{H} \& \mathrm{E}$ (Figure 1).

The poorest results according to the assessment criteria were observed with the decalcified teeth preparations embedded into GMA-based resin and stained with $\mathrm{H} \& \mathrm{E}$. The staining characteristics were found to be highly insufficient and most of them did not allow distinguishing morphological details in this group of histological preparations (Figure 2).

In the preparations which were directly embedded into the MMA-based embedding material without decalcification and stained with H\&E, the quality of revealed morphology of tooth tissues was lower than in the paraffin-embedded decalcified sections but higher than in the decalcified GMA-embedded teeth. However, in the sections of calcified MMA-based embedded teeth we observed low contrast in the staining of cell nuclei and cytoplasm; some morphological 


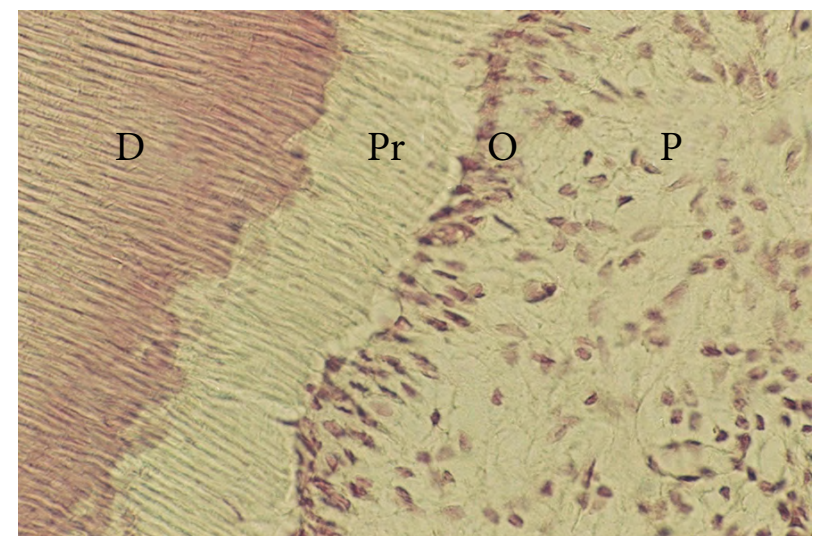

Figure 1. Tooth root decalcified in formic acid, embedded in paraffin, and stained with hematoxylin and eosin (H\&E). Dentin (D), predentin (Pr), odontoblast layer (O), pulp (P). Magnification: $\times 400$

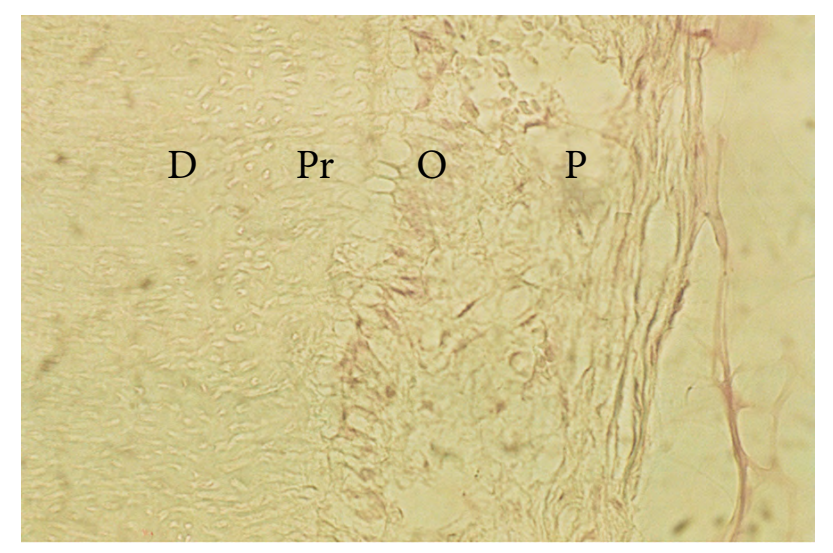

Figure 2. Tooth root decalcified in formic acid, embedded in glycol methacrylate, and stained with H\&E. Abbreviations as for Figure 1. $\times 400$

details, like e.g. the dentin-predentin border, were not distinguishable, and there were wavy artefacts (or unidentified developmental mineralisation details) in dentin (Figure 3).

Statistical analyses (Pearson chi-square test) revealed a highly significant difference $(p<0.001)$ with respect to the assessment criteria in comparison of all groups.

\section{Discussion}

Our findings suggest that the undecalcified tooth preparation prepared by the method used in this study is not more eligible in terms of the histological assessments than the preparations prepared from the tooth which are decalcified in formic acid and embedded in paraffin. According to these results, if decalcification is not a problem in terms of time and study characteristics during the tooth preparation for histological assessment, the most proper method is to

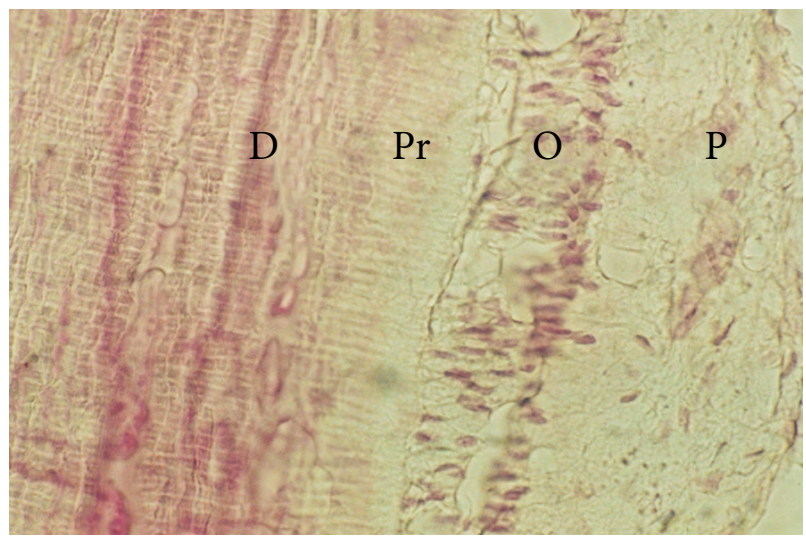

Figure 3. Tooth root embedded in methyl methacrylate -based embedding material without previous decalcification and stained with H\&E. Abbreviations as for Figure 1. $\times 400$

make a histological preparation from the decalcified and paraffin-embedded teeth. It is considered that the decalcification procedures used at the present day makes no significant change in the morphology of microanatomic structures and even in the immunoreactivity $[17,18]$. However, if waiting for the decalcification time is a problem or the study is not suitable for decalcification, the preparations taken from the samples directly embedded into MMA-based embedded material without decalcification and stained with H\&E may also provide acceptable levels of histological details.

Although not commonly used, it was reported that immunohistochemical and histomorphometric studies of MMA-embedded decalcified hard tissues also provided good results [18]. However, this embedding material is more suitable for undecalcified tissues. It was highlighted that MMA could be used in hard tissue immuno- and histochemistry and showed significant improvements in presenting tissue antigenicity [13]. Investigators applied enzyme histochemistry, immunohistochemistry and a wide range of classical histological staining techniques, even in situ hybridisation to hard tissues in which MMA-based embedding resin (Technovit $9100 \mathrm{New}$ ) was used, and reported that good results were produced from all these techniques, especially H\&E staining procedure [9]. However, it has to be noted that this embedding material is especially intended for immunohistochemistry, enzyme histochemistry and in situ hybridisation [15].

It is considered that the routine stains used for paraffin sections provide poor staining in GMA-embedded sections. However, it was reported that optimal morphological integrity and good results with $\mathrm{H} \& \mathrm{E}$ may be obtained also in the GMA-embedded tissues by using modified staining procedures [16]. 
It is noteworthy that the procedure by using GMAand MMA-based resin systems is time-consuming and expensive [19]. Processing steps require changing numerous solvents, preparing a large number of mixtures and special temperatures for each solvent $[11,20]$. Protection from inhalation of harmful gases and strict chemical safety are essential for the safety of the personnel [11]. Additionally, enclosed automatic processors cannot be used for processing and special microtomes with sturdy tungsten carbide knives or metallurgic low-speed diamond saws are required for sectioning [11]. The procedure that will show the result in histological preparation is the final staining. All of the processing steps can increase or decrease the final result of staining quality [21]. The complicated and inconvenient steps in the GMA- and MMA-based resin embedding protocols may negatively affect the staining quality. These procedures become even more expensive, complicated and inconvenient in the enzyme- and immunohistochemical characterization of hard tissue sections processed without decalcification [22]. However, we have shown, that immunohistochemical studies can be successfully conducted in decalcified and paraffin-embedded teeth [23, 24].

Undecalcified bone and tooth sections may be required to analyse mineral distribution. Especially, investigation of calcification areas in pulp or diagnosis of metabolic bone diseases where normal calcification process is impaired may be often necessary. Since the use of GMA- and MMA-based resin embedding systems is often required in the assessment of implanttissue integration, further methodological studies to improve quality of hard tissues visualisation at the microscopic level have to be continued [25]. The results of our study suggest that for routine histochemical assessment and $\mathrm{H} \& \mathrm{E}$ staining of such sections, the MMA-based embedding material may be preferred instead of GMA-based resins.

The histological and histopathological processing of hard tissues for investigational and diagnostic purposes is a routinely-required procedure in the research centres and laboratories of all sizes. The method to be used in this context should be easy-to-apply. However, if decalcified sections have to be used to study hard tissues, and particularly the teeth, paraffin-embedding and $H \& E$ staining is always an easier, more economic and more reliable method than techniques based on embedding the material in resins.

\section{Acknowledgement}

This study was supported by the Research Fund of the Istanbul University (Project No. BYP - 3051). We thank to Dr. Humeyra Kocaelli (Istanbul University
Faculty of Dentistry Department of Oral Surgery and Medicine, Istanbul, Turkey) due to her support for obtaining the materials required for the research.

\section{References}

1. Feuerstein O, Mayer I, Deutsch D. Physico-chemical changes of human enamel irradiated with ArF excimer laser. Lasers Surg Med. 2005;37:245-251.

2. Eimar H, Ghadimi E, Marelli B et al. Regulation of enamel hardness by its crystallographic dimensions. Acta Biomater. 2012;8:3400-3410.

3. Nanci A. Dentin-Pulp Complex. In: Nanci A (ed.). Ten Cate's Oral Histology Development, Structure, and Function. 7th Ed. Louis, Mosby-Elsevier 2008:191-238.

4. Piesco NP. Histology of Dentin. In: Avery JK (ed.). Oral development and Histology. 3rd Ed. New York, Thieme 2002:172-189.

5. Nanci A, Bosshardt DD. Structure of periodontal tissues in health and disease. Periodontol 2000. 2006;40:11-28.

6. Merrell GA, Troiano NW, Coady CE et al. Effects of longterm fixation on histological quality of undecalcified murine bones embedded in methylmethacrylate. Biotech Histochem. 2005;80:139-146.

7. Mukai K, Yoshimura S, Anzai M. Effects of decalcification on immunoperoxidase staining. Am J Surg Pathol. 1986;10:413-419.

8. Mullink H, Henzen-Logmans SC, Tadema TM et al. Influence of fixation and decalcification on the immunohistochemical staining of cell-specific markers in paraffin-embedded human bone biopsies. J Histochem Cytochem. 1985;33:1103-1109.

9. Willbold E, Witte F. Histology and research at the hard tissue-implant interface using Technovit 9100 New embedding technique. Acta Biomater. 2010;6:4447-4455.

10. Bourque WT, Gross M, Hall BK. A histological processing technique that preserves the integrity of calcified tissues (bone, enamel), yolky amphibian embryos, and growth factor antigens in skeletal tissue. J Histochem Cytochem. 1993;41:1429-1434.

11. Karantzoulis V, Liapi C, Papagelopoulos P. Large-scale bone mineral histomorphometry-report of a simplified technique. Histopathology. 2012;61:1174-1182.

12. Baril E, Lefebvre LP, Hacking SA. Direct visualization and quantification of bone growth into porous titanium implants using micro computed tomography. J Mater Sci Mater Med. 2011;22:1321-1332.

13. Yang R, Davies CM, Archer CW et al. Immunohistochemistry of matrix markers in Technovit 9100 New -embedded undecalcified bone sections. Eur Cell Mater. 2003;6:57-71.

14. Singhrao SK, Sloan AJ, Smith EL et al. Technical advances in the sectioning of dental tissue and of on-section cross-linked collagen detection in mineralized teeth. Microsc Res Tech. 2010;73:741-745.

15. Rousselle S, Wicks J. Preparation of medical devices for evaluation. Toxicol Pathol. 2008;36:81-84.

16. Cerri PS, Sasso-Cerri E. Staining methods applied to glycol methacrylate embedded tissue sections. Micron. 2003;34:365-372.

17. Pitol DL, Issa JPM, Caetano FH et al. Decalcification dynamic of dog mineralized tissue by microwaves. Int J Morphol. 2007;25:309-313.

18. Gomes SA, dos Reis LM, de Oliveira IB et al. Usefulness of a quick decalcification of bone sections embedded in methyl 
methacrylate: an improved method for immunohistochemistry. J Bone Miner Metab. 2008;26:110-113.

19. Lim JI, Lee YK. Oleic acid-added embedding medium for histological analysis of hard tissue. Microsc Res Tech. 2009;72:766-771.

20. Mohsin S, O' Brien FJ, Lee TC. New embedding medium for sectioning undecalcified bone. Biotech Histochem. 2006;81:99-103.

21. Troiano NW, Ciovacco WA, Kacena MA. The effects of fixation and dehydration onthe histological quality of undecalcified murine bone specimens embedded in methylmethacrylate. J Histotechnol. 2009;32:27-31.

22. Rammelt S, Corbeil D, Manthey S et al. Immunohistochemical in situ characterization of orthopedic implants on poly- methyl metacrylate embedded cutting and grinding sections. J Biomed Mater Res A. 2007;83:313-322.

23. Keklikoglu N. The localization of Fos B, a member of transcription factor AP-1 family, in rat odontoblasts and pulpal undifferentiated ectomesenchymal cells. Folia Histochem Cytobiol. 2004;42:191-193.

24. Keklikoglu N. Comparison of c-Fos immunoreactivity in pancreatic beta cells and cells with neural crest origin in rats: an immunohistochemical study. Med Hypotheses. 2004;62:537-541.

25. Singhrao SK, Nicholson K, Crean S. Informed choices for challenging specimens when choosing methacrylate resin systems for histology. Microsc Res Tech. 2012;75: $576-585$.

Submitted: 3 October, 2013 Accepted after reviews: 10 January, 2014 